\title{
EL INSTITUTO-ESCUELA DE SEVILLA (1932-1936): UN PROYECTO INCONCLUSO DE RENOVACIÓN PEDAGÓGICA EN ESPAÑA
}

\section{THE INSTITUTE-SCHOOL OF SEVILLE (1932-1936): A PROYECT INCONCLUSIVE OF PEDAGOGICAL RENOVATION IN SPAIN}

http://dx.doi.org/10.15304/ie.28.5488

\author{
Patricia Delgado-Granados \\ Universidad de Sevilla \\ patdelgado@us.es
}

\section{RESUMEN}

Este artículo aborda la labor pedagógica del Instituto-Escuela (I-E) de Sevilla en el periodo comprendido entre 1932 a 1936. Una iniciativa privada llevada a cabo durante la Segunda República que surge en medio del interés social suscitado en Europa en torno a la Escuela Nueva y a las experiencias educativas innovadoras que se estaban llevando a cabo en Europa y EE.UU. Sus objetivos, acorde a los principios pedagógicos de la Institución Libre de Enseñanza (ILE), se orientaron en alcanzar el desarrollo integral de la persona a nivel intelectual, físico, social, moral y artístico. Implicaba una nueva manera de concebir la enseñanza en la que primaba la educación por encima de la instrucción mediante el fomento de valores como el respeto, la justicia, la belleza, la paz, etc. que marcaron una nueva manera de educar. El I-E tuvo un recorrido breve, cruento y claramente diferenciado de los que le procederían. El estallido de la Guerra Civil (1936-1939) y la imposición de la dictadura franquista conllevaron su cierre y posterior expolio. Por lo que el centro sevillano nació y murió con la República. Palabras clave: Instituto-Escuela de Sevilla, modernización, Segunda República española, renovación pedagógica.

\section{SUMMARY}

This article approaches the pedagogic labor of the Institute-School (I-E) of Seville in the period from 1932 to 1936. A private initiative carried out during the Second Republic that arises in the middle of the social interest provoked in Europe concerning the New School and the educational innovative experiences that were carried out in Europe and USA. His aims, chord to the pedagogic beginning of the Free Learning Institution (ILE), were orientated in reaching the integral development of the person to intellectual, physical, social, moral and artistic level. It implied a new way of conceiving education in which education prevailed over instruction by promoting values such as respect, justice, beauty, peace, etc. That marked a new way of educating. Nevertheless, its way was short, bloody and clearly differentiated from those that would proceed. The outbreak of the Civil War (1936-1939) and the 
imposition of the Franco dictatorship led to its closure and subsequent looting. So the sevillian center was born and died with the Republic.

Keywords: Institute-School of Seville, modernization, Spanish Second Republic, pedagogical renovation.

\section{INTRODUCCIÓN}

En las últimas décadas, la historia de la educación ha revindicado como fuente de conocimiento las investigaciones referidas a instituciones educativas y formación docente con objeto de descubrir su capacidad de proyección en las relaciones e interdependencia socio-educativas, contemplando lo local desde una dimensión interdisciplinar (Sola, 1980). En este sentido, el profesor Escolano (2000, p. 22) sugiere que la importancia de la arquitectura escolar no estriba solo en que es:

(...) un programa 'silencioso' de educación, sino la pauta que proporciona al niño las primeras experiencias espaciales y que condiciona el desarrollo de su esquema corporal, así como de ciertas estructuras cognitivas.

Desde esta perspectiva, se concibe el presente trabajo cuyo objetivo es recuperar la historia de una de las instituciones educativas más importantes de la Segunda República, el Instituto-Escuela, desde el ámbito de lo local: la ciudad de Sevilla, cuya andadura se vio truncada con el estallido de la Guerra Civil española (1936-1939) y la instauración de la dictadura franquista, produciéndose su cierre y posterior expolio. No obstante, a pesar de su fugacidad, su encomiable labor educativa como centro "laboratorio" para la formación de profesores y "modelo" de renovación pedagógica, convirtieron al I-E sevillano en referente para conocer algunas de las apuestas de transformación y regeneración educativas llevadas a cabo durante la Segunda República ${ }^{1}$. Ambos términos -centro laboratorio y centro modelo- auguraban nuevos planteamientos pedagógicos basados en los principios de la Escuela Nueva.

El presente trabajo tiene como objetivo recuperar "aquello que ya no existe, que pasó y que ha desaparecido" (Tiana Ferrer, 2007,p. 203), recobrando así una parcela de la historia de las instituciones educativas mediante el análisis del I-E de Sevilla; un proyecto educativo de corte institucionista, lamentablemente inconcluso, cuya proyección se hace vigente, tanto en la formulación del cambio, como en su realización en el marco de un proyecto de renovación pedagógica. Su impronta de cambio y de transformación educativa a través de la unificación de la enseñanza primaria y la secundaria como un todo único y continuo, bajo un planteamiento metodológico de carácter cíclico, basado en un modelo de aprendizaje inclusivo que fomentara el trabajo individualizado y colaborativo, por medio de la orientación y seguimiento continuo del docente, reflejan su proyección actual y su vigencia en el siglo XXI.

Así lo expresaba el Director del Instituto-Escuela de Sevilla, el historiador Juan Mata Carriazo, discípulo de Gómez-Moreno, al señalar su condición como centro de "laboratorio (investigación formativa del profesorado) y estudio (renovación pedagógica)". A.H.U.S. Carpeta Legajo 2600. Informe sobre las actividades del Instituto-Escuela en los meses de enero, febrero y marzo de 1933, p. 2. 


\section{EL PROYECTO EDUCATIVO DE LA SEGUNDA REPÚBLICA: UNA APUESTA A LA MODERNIZACIÓN PEDAGÓGICA}

La Segunda República llegó con el triunfo en las elecciones municipales de abril de 1931 y en un contexto coyuntural de crisis económica internacional, despertando grandes expectativas entre amplios sectores de la población. El esfuerzo modernizador emprendido por el nuevo gobierno se orientó en edificar una nueva sociedad por medio de la reformulación integral del sistema educativo. Por lo que una de las tareas prioritarias fue la reforma de la educación ${ }^{2}$. Francisco Giner de los Ríos (1979, p. 243) expresaba con estas palabras: “(...) de todos los problemas que interesan a la regeneración político-social de nuestro pueblo, no conozco uno solo tan menospreciado como el de la educación nacional".

En efecto, el panorama educativo en la España de principios del siglo XX era devastador. El siglo XIX había dejado un legado en materia educativa lleno de carencias estructurales y debidamente articulado. Las carencias eran aún más evidentes en cuanto a la precariedad de los edificios escolares, la situación de la educación de la mujer, la formación del profesorado o la educación de adultos, etc. Manuel Bartolomé Cossío (1899) denunciaba en tono sarcástico la lamentable situación de la educación española:

(...) hacen bien esos dos millones y medio de niños (que no tienen plaza escolar) en no ir a la escuela, y sus padres obran muy cuerdamente al no enviarlos. Porque si un día se les ocurriese obedecer nuestras sabias leyes, perderían el tiempo y, lo que es más grave, la salud, como pierden ya ambas cosas gran parte de sus aplicados compañeros. Perderían el tiempo, porque no hay en España ni escuelas en que meterlos, aunque fuese almacenados, ni suficiente número de maestros para educarlos de verdad; y perderían la salud, porque los que malamente cupiesen, irían a envenenarse en el pestífero ambiente de unos locales infectos, donde hoy mismo están ya hacinados los niños que asisten (...).

Las tasas de analfabetismo alcanzaban un 32\% entre los mayores de 10 años de edad y, de los veintitrés millones y medio de habitantes, casi seis millones no sabían leer ni escribir. En Sevilla capital el porcentaje de analfabetos mayores de 10 años superaba el $40 \%$ de la población, y sobre el $30 \%$ entre 1920 y 1930; un elevado índice de analfabetismo reflejo de la correlación entre tasas de escolarización y porcentaje de analfabetos.

La profesión docente no solo se hallaba devaluada, sino que vivía en la miseria y existía un déficit de más de veintisiete mil escuelas que se traducía en más de un millón de niños/as sin escolarizar (Pérez Galán, 1988). En palabras del Director General de Enseñanza Primaria, y principal impulsor de la escuela pública nacional en nuestro país, Rodolfo Llopis (1933), se trataba de formar “maestros, buenos maestros", pues constituía una de las piedras angulares del modelo tecnocrático

El programa educativo republicano se inspiró en la ILE, cuya influencia se materializó a lo largo de las tres primeras décadas del siglo XX, promovida por los grupos progresistas que apoyaban una visión modernizadora, laica y democrática de la educación. Su labor pedagógica fue continuada por los llamados "hijos de Giner", entre los cuales destacó su más fiel discípulo, Manuel Bartolomé Cossío. 
de profesionalización docente impulsado por la República. La situación en Andalucía era si cabe aún más precaria y paupérrima, situándose el número de maestros por debajo del promedio español, en torno al 13,1\%3. Como dato representativo en 1930 las provincias andaluzas ocupaban índices inferiores a la media nacional en la proporción de maestros por cada mil habitantes, al igual que en escolarización, encabezando las provincias de Sevilla, Cádiz y Jaén la lista de menor número de habitantes escolarizados. Las crónicas del periodista Luis Bello sirvieron de denuncia desde la prensa de esta situación de precariedad en cuanto a la falta de escuelas en las provincias andaluzas, describiendo los males de la educación para, a partir de ellos, proponer recetas que se implantaron años después durante la Segunda República. La educación debía ser la palanca regeneradora de aquella "enorme masa de gentes iletradas cuya capacidad de cultura - diría Bello- es para nosotros un misterio y para ellos una tragedia" (Bello, 1925). Al proclamarse la República, ésta "tenía que hacer muchas escuelas (...) y verdaderas, especialmente por el espíritu que ha de vivificar la diaria labor docente" (Bello, 2007, p. 18).

Para ello, durante el periodo de 1931 a 1933 se establecieron las líneas básicas de la política educativa republicana dirigidas a incrementar las construcciones escolares y a mejorar las condiciones socio-laborales del magisterio. El primer gobierno republicano en su apuesta firme por la educación, y concretamente por la enseñanza primaria, aprobó un ambicioso plan quinquenal de construcción de escuelas, hasta 27.000 centros escolares. En diciembre de 1932 ya se habían construido 9.620 escuelas, muchas de ellas unitarias. Esta gran inversión en educación no fue estable a lo largo de todo el gobierno republicano (Lorenzo, 2001), y las penurias presupuestarias impidieron alcanzar ese objetivo. El bienio radical-cedista supuso un retroceso en materia educativa traducido en un descenso de las construcciones de escuelas y en la prohibición de la coeducación en las escuelas primarias, medida que se intentó extender a las Escuelas Normales (Lorenzo, 2001). No obstante, los primeros decretos del gobierno republicano reflejaban su especial preocupación en materia educativa, ya que se regularon tres de los temas más relevantes y problemáticos en los últimos años: el bilingüismo, la enseñanza religiosa y la reorganización del Consejo de Instrucción Pública.

Respecto a la enseñanza religiosa en las escuelas, el Decreto de 6 de mayo de 1931 supuso el establecimiento de la libertad de conciencia en la enseñanza, es decir, la laicidad escolar, tomándose una serie de medidas para configurar una "educación nueva”. Con ello, se suprimía la obligatoriedad de la enseñanza religiosa, aunque se mantenía en aquellos casos en los que los padres mostraran su deseo de impartición. En la Revista de Pedagogía de 1931 se sostenía que:

Los educadores españoles estamos, como nadie obligados a ser los defensores más entusiastas de la República. Tenemos el deber de llevar a las escuelas las ideas esenciales en que se apoya: libertad, autonomía, solidaridad, civilidad. Ningún poder puede haber sobre estas ideas; nadie que sea educador puede oponerse a ellas, ya que constituyen también la base de la educación nueva.

Información que recogía la Revista de Pedagogía, en su artículo "Una estadística escolar de España", 25, 1924, pp. 24-26. 
La iniciativa pedagógica del I-E de Sevilla empezó precisamente a desarrollarse en esta etapa de confluencia entre política educativa e institucionismo, bajo el paraguas del primer bienio republicano denominado azañista (1931-1933). En efecto, el contexto de la Segunda República lograría dar respuesta a las exigencias educativas, a la vez que consolidaría las bases de una nueva organización del Estado; un Estado que, ante todo, fuera, como deseaba Manuel Azaña, un "Estado educador" (Aragón, 1974, p. 340). Este compromiso de cambio en la orientación de la política educativa se tradujo en la implantación de un programa abiertamente afín a los principios de la ILE, basado en un modelo estatal de escuela unificada, laica y gratuita, especialmente de la primera enseñanza con medidas de auténtico sabor institucionista, tales como: la obligatoriedad, la coeducación o la abolición de manuales oficiales de texto. Un “(...) periodo de grandes ilusiones, de importantes proyectos y de notables realizaciones” (Puelles, 2007, p. 5).

\section{EL INSTITUTO-ESCUELA DE SEVILLA: UN SOPLO DE AIRE NUEVO A LA CIUDAD}

La Sevilla de los años treinta era una ciudad de fuertes contrastes en el que la educación se encontraba en una situación lamentable: déficits de escuelas, altos índices de analfabetismo, saturación en las aulas, abandono y absentismo escolar ${ }^{4}$. A esta situación de dejación educativa se unía la lacra del caciquismo que controlaba el poder local y mantenía la situación de ignorancia del pueblo. Sevilla era hervidero de contrastes e inquietudes en un ambiente de efervescencia cultural ${ }^{5}$ :

Riqueza acumulada en sectores reducidos de la sociedad, junto a pobreza y miseria infrahumana; alegría festiva con un trasfondo de dramatismo social, que se debatía en conflictos casi permanentes; aparente paz reflejada en el bullicio de los establecimientos públicos (...) justo al lado de situaciones límites, que dividían a la sociedad en bandos irreconciliables. (Salas, 1976, p. 210).

La llegada de la Segunda República supuso un soplo de aire nuevo y de ilusión que se materializaría con proyectos y propuestas pedagógicas innovadoras. En este contexto se sitúa el I-E de Sevilla; que, no siendo una creación específicamente republicana -ya que el primero que se crea fue en Madrid (Real Decreto de mayo de 1918), impulsado por la ILE dentro de un proyecto globalizador-, sí representó una experiencia innovadora de ensayo pedagógico en la Sevilla de los años treinta, nutriéndose pedagógicamente de la Institución (Delgado, 2012).

La creación del I-E de Sevilla, al igual que la de los centros de Valencia y Málaga, vino unida a la disolución de la Compañía de Jesús ${ }^{6}$. Así pues, en cumplimiento con lo marcado por el texto constitucional de 1931, en la tarde del 3 de febrero de 1932 se realizó la incautación oficial del Colegio de los Jesuitas, situado en la Plaza de Villasís, número 6, iniciándose las clases con total normalidad

4 Para una aproximación bibliográfica sobre el contexto social, educativo y político sevillano, consúltense: Álvarez Rey (1993); Corts y Calderón (2006).

5 Cfr. Anuario Estadístico de España. 1930-31 (Luzuriaga, 1926).

6 El artículo 26 de la Constitución de 1931 declaraba la disolución de la Compañía de Jesús, así como de aquellas órdenes religiosas que en sus estatutos incluyeran el voto de obediencia a una autoridad distinta de la legítima del Estado. 
a partir del día siguiente ${ }^{7}$. El nuevo centro funcionó provisionalmente como Colegio Oficial de Primera y Segunda Enseñanza, hasta la creación legal, un mes después, como Instituto-Escuela de Sevilla ${ }^{8}$. En su gestación contó con el apoyo a nivel local del nuevo equipo rector de la Universidad hispalense, representado por Estanislao del Campo y Pedro Castro, junto a otras personalidades cercanas al institucionalismo y al gobierno republicano, como Juan de Mata Carriazo, profesor de la Facultad de Filosofía y Letras y futuro Director del centro. Y, a nivel nacional, con el gobierno del primer bienio, destacando como políticos impulsores Fernando de los Ríos, Rodolfo Llopis y, en especial, el sevillano Domingo Barnés. La gestión educativa del nuevo centro fue encomendada al Patronato de Cultura de Sevilla.

Desde el primer año el cambio de sede se convirtió en una necesidad apremiante por la falta de adecuación del antiguo edificio a las nuevas necesidades formativas ${ }^{9}$. La exigencia de un nuevo local más adecuado que el de la antigua y deteriorada residencia de jesuitas respondía a las características del modelo de enseñanza de la I-E, que requería de recursos e instalaciones diferentes a los que se empleaban en un enfoque puramente academicista y tradicional, tales como la necesidad de espacios amplios al exterior, luminosidad en las aulas, talleres, residencia, etc. El centro elaboró dos propuestas de traslados a edificios - antiguos pabellones construidos con motivo de la Exposición Universal de 1929, el de Argentina o, en su defecto, el de Perú, tal como se refleja en el Informe semestral realizado por la dirección del centro en 1932:

(...) la Exposición Iberoamericana de Sevilla ha dejado algunos (pocos) edificios útiles, hoy sin destino, con los que el I-E se consideraría contento por la excelente adecuación de sus fines. Tales son dos pabellones extranjeros, el de Perú o el de Argentina, ambos en el Parque María Luisa. El pabellón peruano es de construcción más sólida; el de Argentina de distribución más conforme con nuestras necesidades y de más bello emplazamiento, entre el Parque y el Puerto, con mucho sol y bellas perspectivas del río y de la ciudad. Su cesión a España está ya acordada por el gobierno argentino, sólo queda la ratificación del Parlamento. (Rodríguez Bernal, 2006, p. 9).

Finalmente, en la sesión celebrada por el Patronato de Cultura el 4 de octubre de 1932, se acordó proponer al Ministerio de Instrucción Pública, Francisco Barnés, la designación del pabellón argentino como sede del Instituto-Escuela ${ }^{10}$. Propuesta que no llegó a materializarse hasta 1935. Concretamente, el 12 de octubre se produjo la cesión y entrega del pabellón de la República Argentina al Estado español, coincidiendo el día del acto con la celebración de la Fiesta de la Hispanidad. A través de la aprobación de la Orden Ministerial de 8 de octubre se entrega el edificio al I-E "para

7 En cumplimiento del Decreto de 28 de enero de 1932. En su artículo $3^{\circ}$ se otorgan poderes de excepción al Director para que, de manera provisional, seleccionara al equipo docente acorde a los principios de renovación pedagógica de la ILE. A.H.U.S. Carpeta Legajo 2600. Informe sobre las actividades del Instituto-Escuela de Sevilla. Relación nominal de su profesorado, datos para la inspección y Memoria, p. 8.

8 En el Decreto de 2 de marzo de 1932 se perfilan las características básicas del sistema de enseñanza del I-E, apéndice del Real Decreto de 2 de mayo de 1918 que regulaba el de Madrid.

9 A.H.U.S. Carpeta Legajo 2600, Número 1. Informe sobre las actividades del Instituto-Escuela en los meses de octubre, noviembre y diciembre de 1932.

10 Actas del Patronato de Cultura. Sesión de 4 de octubre de 1932, p. 15. 
fines de enseñanza"11, quedando únicamente la tarea de acomodarlo y habilitarlo tras siete años de abandono. Se iniciaba así una nueva etapa prometedora de renovación pedagógica.

No obstante, cuando todo estaba dispuesto para iniciar esta nueva etapa institucional, después de años de lucha por mejorar las condiciones espaciales del centro, estalló el alzamiento militar fascista, encabezado por el general Queipo de Llano. El I-E fue etiquetado como uno de los enemigos de la religión, la civilización y la familia, que, en palabras de Queipo de Llano, se había servido de: “(...) la inteligencia dócil de la juventud y la ignorancia de la masa” para inculcarles "las ideas revolucionarias. Y la triste experiencia de este momento histórico demuestra el éxito del procedimiento elegido por la masonería, el judaísmo y el marxismo"12. Este clima de odio y crispación se visibiliza especialmente en la prensa católica de la época, donde se llega a calificar de "guerra escolar" las disposiciones reformistas en el terreno educativo, utilizándose los ataques a símbolos y edificios religiosos para desprestigiar a sus gobernantes a los que tildan de propiciar el "caos social" "13. El clima de crispación se fue extendiendo por todas las esferas políticas y sociales; y en julio de 1936 se produce el Golpe de Estado y, con ello, la Guerra Civil española que enfrentó al Bando Nacional contra el Bando Republicano. Se inicia así uno de los episodios más cruentos y oscuros de la Historia de España.

La derrota de la democracia truncó brutalmente el proyecto de reforma socio-educativo republicano. La labor del I-E es declarada ilegal, incautándose todos sus bienes y destruyéndose sus instalaciones (aulas, biblioteca, cantina, residencia, laboratorios, talleres, etc.); y, el edificio pasa a destinarse a diversos usos -almacén, dependencias de la Organización Femenina, etc. (Ceba, 1991, p. 6). Se cierra así una etapa de ilusión y compromiso regeneracionista, y se abre otra protagonizada por el expolio, la depuración y el exilio. Los que se quedaron tuvieron que someterse a la censura, la persecución solapada o manifiesta, o al menosprecio de su labor; mientras que los que se marcharon experimentaron las consecuencias del exilio, el trágico desarraigo, el desencuentro consigo mismo... Max Aub (1971, p. 526), en su obra La Gallina ciega, plasma de manera desgarradora el sentimiento del desarraigo, del exilio, de la pérdida de identidad de lo que uno fue y ahora es: “¿Quiénes somos? Tal vez otros (...) ¿Por qué tuerces el alma?, ¿de qué tienes ansia? Sí: te deshaces en deseos, te consume la furia del amor hacia un pasado que no fue, por un futuro imposible".

\section{LA ENSEÑANZA, EL DOCENTE Y SU DIMENSIÓN ÉTICA}

El Instituto-Escuela de Sevilla, de marcada impronta institucionista, tuvo la significación de proyecto piloto de renovación pedagógica para su implementación gradual en las enseñanzas

11 Decreto de Entrega. Orden Ministerial de 8 de octubre de 1935.

12 Bando sobre "Literatura pornográfica y disolvente" firmado por Queipo de Llano, con fecha 4 de septiembre de 1936 (Álvarez Rey, 2006, p. 193). En la obra de Bahamande, Alloucherie y Barbero (2005), se denuncia la detención y fusilamiento de casi todos los profesores del I-E (p. 383). Cabe destacar las similitudes entre este Bando de Queipo de Llano, Jefe del Ejército de Operaciones del Sur, sobre recogida y expurgo de libros, y el Decreto de 7 de diciembre (Boletín Oficial del Estado, 24 de diciembre de 1937).

13 Descalificaciones y anatemas publicados en: El Correo de Andalucía, ABC o La Unión (Álvarez Rey (2006, pp. 17-20). 
sevillanas y de todo el país, junto con los diversos Institutos-Escuelas creados en el resto de España. Para ello, los esfuerzos se centraron en servir como "laboratorio" de ensayo pedagógico, a la vez que "modelo" o centro de referencia a nivel nacional.

De acuerdo a los principios pedagógicos de la ILE, la práctica educativa del I-E se planteó hacia referentes emancipadores con el objetivo de alcanzar la formación integral de la persona a nivel intelectual, físico, social, moral y artístico, desde una dimensión libre y autónoma. Como señala el institucionista Luis de Zulueta (1930: 5) se basaba en una pedagogía de la libertad, aunque:

(...) no tiene nada que ver con el desorden en las clases o la anarquía en los métodos de trabajo. Cada tarea, cada labor lleva en sí su propia disciplina, y cuanto mejor nos ajustemos a ella, tanto más libre será la creación, la obra que resulte.

Un esfuerzo que implicaba primar la educación por encima de la instrucción mediante el fomento de valores como el respeto, la justicia, la belleza, la paz, etc. Este ambiente formador se evidencia curricular y metodológicamente al analizar los recursos pedagógicos empleados para fomentar la comunicación docente-discente, la iniciativa personal, la práctica, el trabajo cooperativo, el deporte, etc., en los que se aboga por una educación, ante todo, humana y no meramente instrumental. Los estudiantes eran los protagonistas de su propio aprendizaje, tomando un papel activo y creativo basado en "enseñar a aprender a hacer las cosas". Para ello, el aula se convertía en taller, el maestro en guía, y los estudiantes en agentes activos. Se trataba de extender el aula a la vida cotidiana, por lo que era frecuente que el aprendizaje se realizara fuera de las aulas, en el exterior. La acción educativa, por lo tanto, no finalizaba dentro del marco reglado, sino que se complementaba y se proyectaba en su entorno, nutriéndose del modelo de enseñanza de su homóloga en Madrid y del Grupo Escolar "Cervantes”, compartiendo con éstos multitud de directrices: “(...) con variaciones de detalle que no alteran la orientación general y aprovechan la experiencia de aquel Centro Modelo", tales como los preceptos pedagógicos institucionista:

(...) el desarrollo mental, la pureza de los sentimientos, la disciplina de la voluntad para formar el carácter, y al propio tiempo el desenvolvimiento del organismo, armónicamente, conseguido éste con el ejercicio de la fuerza, la habilidad en la destreza, y la salud.

Estos preceptos se materializaban en las enseñanzas integradoras, característica básica de su sistema de enseñanza, en cuanto que pretendían abarcar un desarrollo integral del estudiante, no limitándose a las facetas instrumentales o de conocimientos puramente científicos o intelectuales. En consecuencia, un gran número de asignaturas abarcaban temas como la música, historia del arte, visitas a fábricas, iniciación a la cultura técnica, juegos deportivos, etc. Este modelo de enseñanzaaprendizaje, basado en la participación y en el activismo del estudiante, se aprecia en las actividades que realizaban, por ejemplo, con la prensa, el periódico o el teatro. En todas ellas se evidencian elementos potenciadores del activismo pedagógico, tales como el diálogo, las composiciones escritas de temas libres o sugeridos, suprimiéndose los libros de texto. Los contenidos se elaboraban a partir de las explicaciones de los docentes, de las consultas a revistas, libros, prensa, de las excursiones o visitas periódicas a monumentos, museos, etc. Un planteamiento educador que se alejaba por completo de la práctica habitual academicista y, en su lugar, potenciaba otras capacidades tales como la indagación y el descubrimiento, la orientación espacial y temporal, la expresión y argumentación 
de ideas y convicciones, por parte de los estudiantes, etc. Es decir, las experiencias personales y colectivas eran la base para el crecimiento personal e intelectual, partiendo de la relación profesorestudiante y del clima de confianza y respeto donde tenía lugar el diálogo y la escucha activa. Como novedad importante fue emprender en el curso escolar 1932-33 las clases de francés, al igual que se ofertaba un segundo idioma, inglés o alemán, en Secundaria.

Esta metodología centrada en el trabajo personalizado del estudiante y en su aprendizaje cíclico requería una estrecha coordinación y colaboración del equipo docente. El Claustro era, entre otros, uno de los espacios propicios para compartir impresiones, inquietudes y valoraciones, especialmente entre el profesorado del último grado de Primaria y de Secundaria. Esta metodología, centrada en el trabajo personalizado del estudiante y en su aprendizaje cíclico, requería de una estrecha coordinación y colaboración del equipo docente. El Claustro era, entre otros, uno de los espacios propicios para compartir impresiones, inquietudes y valoraciones, especialmente entre el profesorado del último grado de Primaria y de Secundaria.

La evaluación estaba exenta de exámenes y, en su lugar, el estudiante optaba a lo que se denominaba "periodo de revisión" de unos diez días, al cabo de los cuales se le consideraba apto o no apto. No había calificaciones numéricas, sino interpretaciones y observaciones de tipo cualitativo, relacionadas con la conducta, motivación o actitud del estudiante. Tampoco realizaban examen de ingreso para acceder a la Enseñanza Secundaria los que procedían de la Primaria del centro ${ }^{14}$.

En el curso inicial (1932-33) las enseñanzas se estructuraron en: tres grados de Sección Primaria con alumnos de seis a once años de edad, aproximadamente, y en grupos reducidos para llevar un mejor seguimiento individual de cada niño, concebido como eje del proceso educativo, siguiendo así los modelos roussoniano de la ILE y de la Escuela Nueva ${ }^{15}$. En la Primaria se siguió principalmente el modelo del Grupo Escolar Cervantes, mientras que en la Secundaria el modelo de su homóloga en Madrid. Por lo tanto, el proyecto pedagógico venía avalado por las experiencias positivas de ambas instituciones madrileñas. Para ello, la dirección del centro, secundado por el Claustro de profesores, tuvo especial empeño en reforzar esta compenetración entre ambas secciones para "evitar la disociación que existe entre las del I-E de Madrid", colaborando unos con otros. Así, por ejemplo, en el curso académico 1932-33, el profesor de primaria y secretario, Víctor Navarro Pavía, inició unas clases de caligrafía en todos los cursos de la sección de secundaria. Asimismo, los alumnos de ambas secciones recibían por igual enseñanzas de francés, Cultura Física y Juegos (Deportes) y Música (Canto Coral). El objetivo no era otro que potenciar la compenetración y

14 A.H.U.S. Carpeta Legajo 2600. Informe sobre las actividades del Instituto-Escuela durante los meses de octubre a diciembre de 1932, p. 5.

15 Del movimiento de Escuela Nueva, destacarían, entre otras, las ideas y principios pedagógicos de John Dewey que sirvieron de fuente de inspiración para la transformación social que pretendía tanto la ILE como otros grupos progresistas, especialmente a partir de los años veinte del siglo XX. En todo caso, es preciso señalar que el temprano interés de Giner de los Ríos por la obra del pedagogo y filósofo norteamericano ya se puso de manifiesto en sus escritos educativos, realizados en las últimas décadas del siglo XIX. Desde entonces, Giner combinó el modelo educativo deweyano, con las ideas educativas ¡de Fröbel y la filosofía de Krause, como parte fundamental de su propuesta pedagógica (Díaz, Pita y Terradillos, 2000). 
proporcionalidad de los dos niveles educativos para que el paso de la primera a la segunda enseñanza se realizara con la menor violencia posible y, por otro lado, evitar que el centro se convirtiera en:

(...) un Instituto con escuela preparatoria, de cuyas tareas se encuentren despreocupados los profesores de la secundaria como ajenos a los afanes de los de la Primaria; pero, también que la escuela crezca desmesuradamente, de suerte que el Instituto resulte simplemente apéndice suyo que ni siquiera baste recibir alumnos que aquella prepare. Ambos riesgos son perfectamente posibles, y en ellos han caído otros establecimientos de la enseñanza oficial, en cuya experiencia aprende el Instituto Escuela de Sevilla ${ }^{16}$.

La selección del personal docente se realizaba mediante un cursillo selectivo ofertado en el Colegio Cervantes. Este procedimiento de selección docente fue considerado por la institución sevillana "un rotundo acierto" 17 . Además, el profesorado contaba con la colaboración de un grupo de jóvenes estudiantes de los últimos años de universidad, que asistían a las clases asiduamente con objeto de conocer las innovadoras metodologías y estudiar de manera directa y presencial el sistema del Instituto-Escuela, sin percibir remuneración alguna solamente acompañaban al profesorado y colaboraban en su tarea educativa.

El planteamiento educativo que impregnaba la praxis pedagógica de los profesores y las materias se aprecia en la propia metodología. Para ello, se diseñó un plan de enseñanza de carácter cíclico conforme a la normativa curricular nacional, pero con la autonomía suficiente que permitiera ampliar el currículum con objeto de fomentar el estudio práctico y directo de la naturaleza, la lectura, la convivencia, el esfuerzo motivado, la curiosidad, la iniciativa, el debate, la lectura, etc. Así la enseñanza del lenguaje, la geografía, la historia, las ciencias naturales o las matemáticas, de uno y otro nivel, se acompañaban de ejemplos de lecciones elaboradas por el profesorado, reflejo de su calidad científica y humana. En este sentido, la formación pedagógica del docente fue uno de los aspectos más interesantes del Instituto-Escuela sevillano. Su director, el Catedrático de Historia del Arte y Excursiones, Juan de Mata Carriazo Arroquia, en la línea de pensamiento de Giner de los Ríos, estaba convencido de que las transformaciones y renovaciones en educación debían empezar previamente por el cambio en la práctica educativa de los docentes ${ }^{18}$. El proceso de aprendizaje activo, centrado en el estudiante y estrechamente relacionado con su contexto vital, requería de un profesorado altamente motivado y conocedor de las metodologías innovadoras. En consecuencia, se ofertaban actividades de perfeccionamiento docente a través de visitas y estancias a instituciones similares en el extranjero interesadas por la renovación pedagógica y con modelos formativos más avanzados pedagógicamente. En este caso, el Director del I-E sevillano, Juan de Mata Carriazo, con pensión de la Junta para Ampliación de Estudios, realizó en el curso 1935-36 diversas estancias formativas en Italia, Francia, Suiza e Inglaterra, donde visitó “monumentos y museos”; y publicó

16 A.H.U.S. Carpeta 2600. Informe sobre las actividades del Instituto-Escuela de Sevilla durante el curso 1934-35, p. 2.

17 Ibídem, p. 3.

18 Juan de Mata Carriazo había sido profesor aspirante en el I-E de Madrid, por lo que conocía de primera mano que, en cualquier proyecto de reforma educativa, la formación docente era una pieza clave.

19 A.H.U.S. Carpeta Legajo 2600. Informe sobre las actividades del Instituto-Escuela durante los meses de octubre a diciembre de 1932, p. 6. 
en el Boletín l'Office International des Intituts d'Archéologie et d'Histoire de l'Art, su trabajo sobre Les fouilles d'Italica. Por su parte, el profesor de la sección Primaria, Víctor Navarro Pavía, inició en estos años la impresión de la serie de libros para el I-E, bajo el título general de "Esquemas de trabajos", en colaboración con el profesor Mengo, del Grupo Escolar "Cervantes" de Madrid" Asimismo, fueron frecuentes las asistencias e intervenciones de profesores del centro a reuniones internacionales de carácter pedagógico celebradas a nivel nacional e internacional, tales como los Cursos de la Universidad Internacional de Verano de Santander organizados por la Universidad de Liverpool, etc. ${ }^{20}$

\section{LAS COLONIAS Y LAS EXCURSIONES ESCOLARES: UNA NUEVA FORMA DE APRENDER}

Algunas de las iniciativas que alcanzaron más popularidad en el I-E fueron, sin duda, las colonias y las excursiones escolares que permitían salir a nuevos espacios formativos rompiendo con el encuadre pedagógico tradicional, para en su lugar adoptar un nuevo enfoque de aprender mediante el método intuitivo y experiencial. A la vez se daba respuesta a las nuevas experiencias pedagógicas e higienistas, dirigidas al fomento del ejercicio físico, al contacto con la naturaleza y a la convivencia entre profesorado y estudiantes, en un contexto distinto al reglado, y potenciando el desarrollo integral de las personas a nivel físico, cultural y moral.

La primera, las colonias escolares, tenían una finalidad de carácter fisiológico-caritativo, pues se concebían como un espacio residencial durante las vacaciones estivales para que los niños se robustecieran dando respuesta a las experiencias higienistas del último cuarto del siglo XIX y proyectadas por la ILE. Su organización corría a cargo del equipo docente, contando con la colaboración del médico del I-E, José Moreno Cenicero, encargado de la confección de fichas y de los exámenes clínicos de todos los alumnos ${ }^{21}$. Este servicio médico elaboraba una ficha por estudiante indicando las revisiones periódicas y los "niños necesitados de los beneficios de los baños de mar y colonias", distribuyéndose en tres categorías, de mayor a menos necesidad ${ }^{22}$. En este sentido, un problema que se planteó desde sus inicios fue la selección de los alumnos que debían asistir a las colonias. Por lo que se designó a una comisión organizadora elegida por el Claustro, y formada por los profesores que iban a asistir a las mismas. Los motivos que aludían los padres para que sus hijos fueran incluidos en las expediciones eran, en la mayoría de los casos, por temas de salud, tal como

20 Como fue el caso del profesor y secretario del I-E, Alfredo Malo Zarco, Catedrático de Castellano y Literatura. A.H.U.S. Carpeta Legajo 2600. Informe sobre las actividades del Instituto-Escuela de Sevilla durante el segundo semestre de 1935, pp. 5-6.

21 Así, por ejemplo, en el curso académico 1935-36, acompañaron a los estudiantes, los siguientes profesores: José Bernal Ulecia, Catedrático de Geografía e Historia; Manuel Berniel Luque, Maestro Nacional de la Sección Primaria; y, Ana María Robles García, Profesora Aspirante de la Sección de Letras. A.H.U.S. Correspondencia, presupuestos y oficios de la Colonia veraniega del Instituto-Escuela de Sevilla durante el año 1934, p. 17.

22 bidem, p. 17. 
se refleja en la correspondencia dirigida al director del I-E, por parte de una madre en la que muestra su preocupación por la falta de robustez de su hijo: “(...) tengo entendido que los baños de mar deben ser tres consecutivos Ruego sea incluido mi hijo en la Colonia por considerarlo de necesidad por falta, a mi parecer, de robustez". Para las familias que carecían de recursos económicos se contemplaba su asistencia de manera gratuita, los llamados “colonos gratuitos". Había, por tanto, un factor compensatorio atendiendo a niños de constitución más débil y de familias humildes.

La primera colonia escolar se realizó en el verano de 1933, por iniciativa de su profesorado que participaba en las mismas, como una proyección educativa del I-E en los meses estivales, y contó con la colaboración de la "Asociación de Amigos del Instituto-Escuela" como institución fraterna $^{23}$. Se propuso la Finca La Inmaculada Concepción, emplazada en las inmediaciones del Puerto de Santa María, en plena bahía de Cádiz, y cedida temporalmente a estos efectos por el Patronato administrador de los bienes incautados a la Compañía de Jesús ${ }^{24}$. El proyecto fue acogido con entusiasmo por los familiares de los alumnos, realizándose meses más tarde la petición de cesión de la finca como colonia del I-E de Sevilla. La residencia se componía de una antigua casa de una planta que contaba con setenta y siete camas. Se solía hacer compra todos los días de leche, pan, verduras, frutas, carne o pescado, queso, dulce de membrillo, entre otros, con objeto de que tuvieran una dieta equilibrada. El objetivo, además de sanitario y físico-corporal mediante el fortalecimiento y mejora de la salud, era también educativo, pues mediante un ambiente jovial, a la vez que "disciplinado y de orden se conseguía la confianza, el reconocimiento y el cariño hacia sus profesores y compañeros" 25 .

La segunda iniciativa, las visitas y las excursiones, también fueron referente en el I-E de Sevilla, ya que se partía del convencimiento pleno de las magníficas posibilidades que permitía la realización de la acción educativa al aire libre en lugar del recinto de la clase, del taller o del campo de juegos, potenciando la convivencia y el aprendizaje espontáneo de los estudiantes. Con "plena meditación" por parte del grupo de profesores las excursiones evitaban un plan fijo y cerrado en horario y forma permitiendo, en su lugar, la improvisación, la espontaneidad y la motivación hacia lo desconocido. Cada excursión tenía un objetivo sobre el que se procuraba interesar por adelantando a los alumnos, teniendo igual importancia "las insospechadas lecciones ocasionales", pues:

(...) el ideal de enseñanza es mostrar a los niños las cosas no en esquemas o descripciones aisladas cada una de las demás, sino en la realidad misma, con la eficacia de su directa contemplación y estudio, y del análisis de sus relaciones recíprocas ${ }^{26}$.

Las visitas a monumentos, museos, campos, fábricas y talleres, así como las excursiones a pueblos y ciudades, partían de una metodología sistemática de enseñanza integrada en el currículo,

23 A.H.U.S. Carpeta Legajo 2600. Datos para la Inspección, p. 3

24 Actas del Patronato de Cultura, Sesión de 17 de junio de 1933, p. 26.

25 Instituto-Escuela de Sevilla. Colonia Veraniega de 1935. 15 de junio a 14 de septiembre. Folleto imp. Mejías. Sevilla.

26 A.H.U.S. Carpeta Legajo 2600. Informe sobre las actividades del Instituto-Escuela durante los meses de octubre a diciembre de 1932, p. 7. 
pudiéndoseles dar el carácter de enseñanzas -"Historia del Arte", "Excursiones", etc.-, pues estaban planteadas en el horario semanal. En este sentido, a través de los Informes del centro, se constata el interés del I-E por visitar principalmente lugares apartados o desconocidos, monumentos inéditos de pueblos modestos, en los que difícilmente tendrían los alumnos ocasión de visitar y “(...) solo más tarde, para que los puedan saborear con verdadero conocimiento, visitan los alumnos ciudades importantes y monumentos capitales"27. En el curso académico 1935-36 se estudió la posibilidad de que alumnos de cursos superiores realizaran excursiones y visitas a lugares más lejanos. No obstante, esta posibilidad no llegó nunca a materializarse tras el alzamiento fascista.

\section{CONCLUSIONES}

Numerosos estudios, desde los clásicos (Delors, 1996; Faure, 1972) hasta los más recientes (MECD, 2016; OCDE, 2016), han resaltado los beneficios de un adecuado aprovechamiento de la escolarización, tanto a nivel social, como cultural y económico. El I-E de Sevilla, a pesar de las circunstancias internacionales de crisis económica en el que se contextualizó, es un ejemplo claro, a considerar, de aprovechamiento de la escolarización al tener como finalidad educativa el desarrollo integral del individuo en todas sus esferas -cognitiva, emocional, física, social, moral, etc.-. Su proyección actual se manifiesta, por tanto, en el carácter renovador de sus enseñanzas, bajo un planteamiento globalizador e integrador que va desde la educación artística, física, actividades al aire libre, hasta las materias instrumentales, sobre todo el lenguaje como soporte esencial de la comunicación. Una concepción renovadora de la enseñanza que rompía con el modelo tradicional de escuela, dejando atrás el marco del aula como exclusivo ámbito curricular.

Han pasado más de setenta y cinco años de la creación del I-E de Sevilla, sin embargo, las medidas institucionistas de renovación pedagógica que se llevaron a cabo podrían materializarse en la actualidad. Su metodología tendría hoy día plena vigencia en cuanto al planteamiento cíclico de las enseñanzas, el modelo de aprendizaje inclusivo, el fomento de un trabajo individualizado bajo el seguimiento u orientación continua del docente. A pesar de su corta trayectoria, no se puede negar su relevancia y vigencia en cuanto a que muchos de los procedimientos educativos, están presentes en la escuela actual.

\section{REFERENCIAS BIBLIOGRÁFICAS}

Algora, C. (1996). El Instituto-Escuela de Sevilla (1932-1936). Una proyección de la Institución Libre de Enseñanza. Sevilla: Diputación Provincial de Sevilla.

Álvarez Rey, L. (1993). La derecha en la II República: Sevilla 1931-1936. Sevilla: Servicio de Publicaciones de la Universidad de Sevilla.

Álvarez Rey, L. (Coord.) (2006). Andalucía y la Guerra Civil. Estudios y perspectivas. Sevilla: Diputación de Sevilla.

27 A.H.U.S. Carpeta Legajo 2600. Informe sobre las actividades del Instituto-Escuela durante los meses de octubre a diciembre de 1932, pp. 7-8. 
Añón, R. Ma (2005). La arquitectura de las escuelas primarias municipales de Sevilla. Sevilla: Servicio de Publicaciones Universidad de Sevilla.

Aragón, M. (1974). Estudio Preliminar Azaña M. La velada en Benicarló. Diálogo de la guerra de España. Madrid: Editorial Castalia.

Aub, M. (1971). La Gallina ciega. Dietario español. México: Joaquín Mortiz.

Bahamonde, A., Alloucherie, J. y Barbero, E. (2005). Un año con Queipo de Llano (Memorias de un nacionalista). Seguido de Noches de Sevilla y de El infierno azul. Madrid: Espuela de Plata.

Bello, L. (1925). Anteproyecto. Para una Sociedad de Amigos de la Escuela. II. Los medios, El Sol, 9 de diciembre.

Bello, L. (1926). Viaje por las escuelas de España. Madrid: Magisterio Español.

Bello, L. (2007). Viajes por las Escuelas de Andalucía. Colección los viajeros. Sevilla: Renacimiento, Centro de Estudios Andaluces.

Ceba, J. J. (1991). Instantes de paz en la guerra. Sevilla: Junta de Andalucía.

Corts, Ma I. y Calderón, C. (Coords.) (2006). Estudios de Historia de la Educación en Andalucía. Sevilla: Servicio de Publicaciones de la Universidad de Sevilla.

Cossío, M. B. (1899). La reforma escolar. Revista Nacional, 31 de octubre, pp. 321-323.

Delgado, P. (2012). Esbozo histórico de la influencia pedagógica de la Institución Libre de Enseñanza en Andalucía. En J. García-Velasco y A. Morales Moya (Eds.), La Institución Libre de Enseñanza y la cultura española, Vol. 2. Madrid: Fundación Francisco Giner de los Ríos y Acción Cultural Española, pp. 360-369.

Delors, J. (1996). La educación encierra un tesoro. Ediciones UNESCO.

Díaz, E., Pita, J. M. y Terradillos, J. (2000). Jornadas homenaje a Giner de los Ríos. Jaén: Universidad de Jaén.

Escolano, A. (2000). Tiempos y espacios para la escuela. Madrid: Biblioteca Nueva.

Escolano, A. (Dir.) (1992). Leer y escribir en España. Doscientos años de alfabetización. Madrid: Fundación G.S. Ruipérez.

Esteban, L. y Mayordomo, A. (1984). El Instituto-Escuela de Valencia 1932-1939. Una experiencia de renovación pedagógica. Valencia: Universidad de Valencia.

Faure, E. (1972). Aprender a ser. La educación del futuro. UNESCO.

Giner de los Ríos, F. (1900). El problema de la educación nacional y las clases productoras. BILE, 478, pp. 1-8.

Giner de los Ríos, H. (1979). Preceptos pedagógicos para el profesorado de las Escuelas libres, neutrales o laicas de niños y niñas recomendados por Hermenegildo Giner de los Ríos. Reedición con un prólogo introductorio de Buenaventura Delgado, Barcelona.

Jiménez-Landi, A. (1996). La Institución Libre de Enseñanza y su ambiente. Periodo de expansión influyente. Tomo IV, Madrid: Taurus.

Juan Borroy, V. (2013). El Magisterio como agente de innovación pedagógica (1900-1936). Innovación educativa, 23, pp. 97-109.

Juan Borroy, V. (2004). La tarea de Penélope. Cien años de escuela pública en Aragón. Zaragoza: IFC, Biblioteca Aragonesa de Cultura.

Lacomba, J. A. (Coord.). Represión y muerte en la Andalucía del 36. Dossier publicado en Diario-16 Andalucía, 11 de agosto de 1986, pp. 2-6. 
Llopis, R. (1933). La revolución en la escuela. Dos años en la Dirección General Primera Enseñan$z a$. Madrid: M. Aguilar Editor.

Lorenzo, J. A. (2001). Claves históricas y educativas de la Restauración y de la Segunda República (1876-1936). Revista Complutense de Educación, 12(1), pp. 215-250.

Luzuriaga, L. (1926). El analfabetismo de España. Madrid: J. Cosano (Museo Pedagógico Nacional), $2^{\mathrm{a}} \mathrm{Ed}$.

Marrou, H. I. (1999). Del conocimiento histórico. Barcelona: Idea Universitaria.

OCDE (2016). Panorama de la educación. OCDE.

Otero, E. (2003). La irrupción de la Pedagogía en la universidad española: Manuel Bartolomé Cossío en la Cátedra de Pedagogía Superior. Revista de Educación, 332, pp. 249-263.

Palacio, L. (1988). Instituto-Escuela. Historia de una renovación educativa. Madrid: Servicio de Publicaciones del Ministerio de Educación y Ciencia.

Pérez Galán, M. (1988). La enseñanza en la Segunda República. Madrid: Mondadori.

Pozo, Ma M. del (2008). Educación para la ciudadanía democrática en la Segunda República: un intento de construcción de la identidad nacional de la escuela. Revista de Historia de la Educación, 27, pp. 105-135.

Puelles, M. de (1980). Educación e ideología en la España contemporánea. Barcelona: Labor.

Puelles, M. de (2007). Política y educación en la España contemporánea. Madrid: UNED.

Ramírez, E. (1994). La formación inicial del profesorado en el Instituto-Escuela, Espacio, Tiempo y Forma, Serie V, $\mathrm{H}^{\text {a }}$ Contemporánea, T. 7.

Rodríguez Bernal, E. (2006). La Exposición Ibero-americana de Sevilla. Sevilla: Ayuntamiento de Sevilla, Icas.

Salas, N. (1976). Sevilla: Crónicas del siglo XX. Tomo II (1921-1940). Sevilla: Universidad de Sevilla.

Sola, P. (1980). Nuevas corrientes en historia de la educación. Cuadernos de Pedagogía, 65, pp. 66-69.

Tiana Ferrer, A. (2007). Memoria histórica y sistema educativo. En: Aróstegui, J. (Ed.). España en la Memoria de tres generaciones. De la esperanza a la reparación. Madrid: Editorial Complutense y Fundación Francisco Largo Caballero, pp. 203-218.

Viñao, A. (2000). Un modelo de reforma educativa: los institutos-escuelas (1918-1936). Boletín de la Institución Libre de Enseñanza, 39, pp. 63-88.

Zulueta, L. de (1930). La pedagogía de la libertad. Revista de Pedagogía, 97, pp. 1-20. 